L'article de la Prof. Valerie Junod relate un changement de pratique important du Tribunal fédéral. Le nouvel arrêt améliore clairement la situation du médecin attaqué, qui pourra dorénavant se défendre en toute connaissance de cause.

Cependant, un point reste insatisfaisant: le médecin est toujours tenu de fournir la preuve que sa clientèle est différente de celle de la moyenne de ses collègues. Or, la littérature consacrée au managed care aux Etats-Unis montre depuis de nombreuses années déjà que les patients ne sont généralement pas répartis de manière homogène entre les médecins d'une discipline particulière [1]. Si on ne tient pas compte des statistiques en matière de morbidité, les procédures actuelles sont une invitation plutôt cynique à l'endroit du médecin, l'incitant à sélectionner ses patients en fonction du risque économique qu'ils représentent. L'article du Dr Michel Romanens, lui, démontre clairement que I'indice ANOVA ainsi que la méthode utilisée pour calculer la moyenne des coûts, tels qu'ils sont employés par santésuisse à I'heure actuelle, comportent d'importantes faiblesses sur le plan juridique et statistique, et qu'ils ne permettent pas de déceler des pratiques non économiques de la part de médecins. Dans son article, l'auteur établit une synthèse des principaux résultats de trois expertises juridiques et statistiques mandatées par I'Association VEMS (éthique et médecine), il revient sur la dernière jurisprudence du Tribunal fédéral concernant la procédure d'économicité, et il présente des perspectives d'avenir. santésuisse est invitée à élaborer de nouvelles bases pour les procédures EAE, conjointement avec la FMH. Et le Parlement est prié de compléter l'art. 56 de la LAMal, de façon à ce que la tâche de développer une procédure d'économicité adéquate et digne d'un Etat démocratique soit confiée aux caisses-maladie et aux associations de fournisseurs de prestations.

Hanspeter Kuhn, avocat;

Gabriela Lang, avocate; Service juridique de la FMH

1 Voir notamment: Kongstvedt PR. Essentials of Managed Health Care. $4^{\text {th }}$ edition. Gaithersburg: MD Aspen Publication; 2001.

P. 400: Peer group, adjusted for age, sex and case-mix/severity of illness - «the issue of severity must be addressed».

\title{
Polypragmasie: enfin une bonne nouvelle
}

Valerie Junod

Professeur de droit aux Universités de Genève et Lausanne

L'auteur remercie Betty et Charles-André Junod pour leurs commentaires critiques.

Correspondance:

Prof Dr Valerie Junod Université de Lausanne - HEC Internef bureau 615 CH-1015 Lausanne Tél. 0216923428 Fax 0216923305

valerie.junod@unil.ch

Anticipant Noël de quelques jours, un arrêt du Tribunal fédéral (TF) apporte un peu de baume au cœur des médecins [1]. En effet, il limite le pouvoir des caisses et de santésuisse (l'association faîtière) de contrôler et sanctionner l'(in)économicité des professionnels de la santé [2], en première ligne bien évidemment les médecins.

Depuis déjà 1969, les caisses exigent des médecins qu'ils remboursent le «trop-perçu». Le montant réclamé est calculé par rapport à la moyenne de leurs collègues exerçant dans le même lieu et la même spécialité [3]. Selon la jurisprudence, une marge de tolérance de 30\% environ est accordée. Par exemple, le cardiologue lausannois qui facture à l'assurance-maladie des soins directs (traitements) et/ou indirects (notamment médicaments et analyses) pour un montant par patient supérieur de plus de $30 \%$ que la moyenne de ses collègues cardiologues vaudois peut se voir

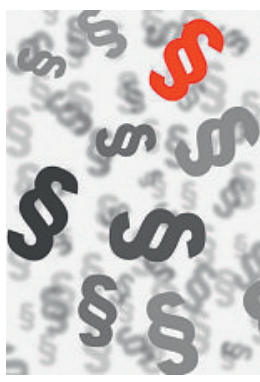
réclamer le paiement de la différence [4]. Pour échapper à l'application de cette méthode dite «statistique», le médecin peut tenter de faire valoir des justifications objectives (par ex. la composition inhabituelle de son collectif de patients). C'est cependant à lui d'en apporter une preuve stricte. Après un ou plusieurs avertissements, les caisses, qui se fondent sur les statistiques de santésuisse, peuvent décider d'entamer une action en paiement devant un tribunal cantonal. Quelques 40 procès étaient pendants en 2007 au travers la Suisse [5].
Dans l'affaire ici en cause, un généraliste genevois s'était fait condamné à payer près de 300000 francs. Recourant contre le jugement du Tribunal arbitral genevois, le médecin élevait différentes griefs. La plupart ont été rejetés - brièvement et assez sèchement [6]. Cependant, l'un d'eux a été retenu. Et il revêt une grande importance pratique. Le médecin invoquait une violation de la Constitution (art. 29) et de la Convention européenne des droits de l'homme (art. 6). Ces dispositions garantissent le droit d'être entendu. Plus particulièrement, le défendeur considérait que les chiffres, sur lesquels s'était basé le juge sos cantonal, étaient opaques au point de l'empêcher d'exercer STS 1 valablement ses droits de la dé11 fense. Rappelons que santésuisse S5 fournit au juge le montant des S 18 dépenses occasionnées par le 103 médecin attaqué en justice \$ 3 ainsi qu'une moyenne de celles des médecins du groupe de comparaison. Complétant cette moyenne, figurent «le nombre de médecins pris en considération et l'âge moyen des patients traités par ceux-ci». Or, s'agissant précisément des données du groupe de contrôle, le TF arrive à la conclusion que celles-ci sont trop lacunaires pour pouvoir être comprises, contrôlées et critiquées par le médecin défendeur. Le TF prône donc «un accès plus étendu». Quel doit-il être? Le TF apporte deux précisions:

«D'une part, seule la connaissance du nom des médecins composant le groupe de référence permet effectivement de vérifier si des praticiens particuliers 
figurent dans la liste alors qu'il appartiennent à un autre groupe ou si d'autres praticiens ne figurent pas dans la liste alors qu'il devraient s'y trouver. D'autre part, seule la connaissance, sous forme anonymisée, de la répartition des coûts pour chaque médecin du

\section{Cet arrêt donne au médecin l'opportunité d'expliquer ses coûts supérieurs par des différences concrètes avec} un ou plusieurs de ses collègues.

groupe de comparaison, à savoir la connaissance des mêmes données anonymisées que celles produites par santésuisse le concernant pour chacun des médecins du groupe mentionné («données du pool de données santésuisse»), permet au praticien contrôlé de se situer concrètement par rapport à ses confrères et d'être mieux à même de produire une défense ciblée et pertinente.» [7].

En résumé, le TF ordonne à santésuisse de fournir au médecin attaqué l'identité des médecins formant le collectif de comparaison. L'association faîtière doit aussi fournir, pour chacun d'entre eux, des données équivalentes à celles concernant le médecin défendeur (à savoir, pour les coûts directs: «coûts de traitements prodigués et de médicaments fournis, nombre de visites à domicile et de consultations; âge moyen et nombre de malades; totaux et indices déduits de ce qui précède» [8]; pour les coûts indirects «coût des médicaments, analyses et séances de physiothérapie prescrits; totaux et indices déduits de ce qui précède» [9]).

Indubitablement, pour le médecin défendeur, cet arrêt représente une excellente nouvelle. Elle lui donne l'opportunité d'expliquer ses coûts supérieurs par des différences concrètes avec un ou plusieurs de ses collègues. Par exemple, il peut expliquer que certains médecins du groupe de comparaison n'exercent pas ou plus la même spécialité médicale; il peut plus facilement mettre en lumière des spécificités de sa clientèle par rapport à celles de certains collègues. Ce n'est pas une panacée, mais l'égalité des armes s'en trouve améliorée.

Pour les autres médecins, cette transparence peut paraître gênante. Il n'est pas exclu que, des données désormais exigées, le médecin poursuivi puisse déduire le revenu de ses collègues [10]. Le TF n'a pas dit comment le droit d'être entendu devra être concilié avec le droit au respect de la vie privée et la protection des données.

Enfin, pour les caisses, cet arrêt signifie qu'elles ne peuvent maintenir un monopole sur les données qu'elles exploitent pour prouver une polypragmasie. Si elles veulent obtenir la condamnation d'un médecin, elles doivent consentir une transparence nettement accrue. Il est bien possible qu'à terme tout le processus de contrôle d'économicité s'en trouve altéré.

\section{Références}

1 Arrêt du 15 décembre 2010, référence 9C_968/2009, disponible sur Internet à http://jumpcgi.bger.ch/ cgi-bin/JumpCGI?id=15.12.2010_9C_968/2009

2 A l'origine, le terme «polypragmasie» était utilisé. Il a été petit à petit remplacé par l'expression «contrôle de l'économicité». Ce contrôle se fonde sur l'art. 56 de la Loi fédérale sur l'assurance-maladie. Pour une présentation complète du système de contrôle et de ses forces et faiblesses, voir V. Junod. Polypragmasie, analyse d'une procédure controversée. Dans: Cahiers genevois et romands de sécurité sociale. $\mathrm{N}^{\circ} 40 ; 2008$. p. 140 ss.

3 Le groupe de comparaison doit compter au minimum dix médecins. Cf. considérant 5.3 .

4 Comme la réclamation porte également sur les coûts indirects, c'est-à-dire des frais que le médecin n'a pas lui-même perçu, il ne s'agit pas à proprement parler d'un remboursement.

5 santésuisse. Les évaluations d'économicité de santésuisse. 2009 ; p. 9.

6 Cf. considérant 2.3 (argument de la péremption); considérant 3.2 (argument de la qualité pour agir et du montant réclamé par chaque caisse); considérant 6.2 (argument du bien-fondé de la méthode statistique; «Le Tribunal fédéral admet depuis longtemps le recours à la méthode statistique comme moyen de preuve permettant d'établir le caractère économique ou non des traitements prodigués par un médecin donné et n'entend pas modifier sa pratique.»).

7 Arrêt cité, considérant 6.3.3.

8 Arrêt cité, considérant 6.3.2.

9 Id.

10 Les coûts directs et indirects des médecins du groupe de comparaison doivent être fournis individuellement, mais de manière anonyme. Cependant, en recoupant ces données avec la liste nominative de ces médecins, il est possible que le défendeur puisse les attribuer à des médecins individuels. Le risque est d'autant plus élevé que le collectif de comparaison est réduit (pour rappel, un minimum de 10 médecins). 